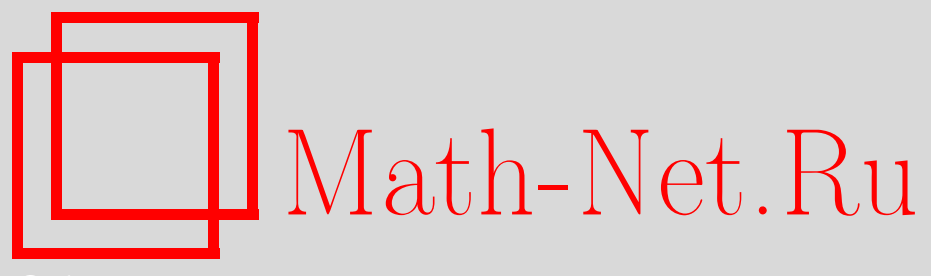

П. Н. Ярыкин, Поведение нелинейного случайного процесса в окрестности его стационарных распределений, УМH, 2006, том 61, выпуск 4, 199-200

DOI: https://doi.org/10.4213/rm1727

Использование Общероссийского математического портала Math-Net.Ru подразумевает, что вы прочитали и согласны с пользовательским соглашением http://www.mathnet.ru/rus/agreement

Параметры загрузки:

IP : 54.162 .85 .209

26 апреля 2023 г., 12:49:53

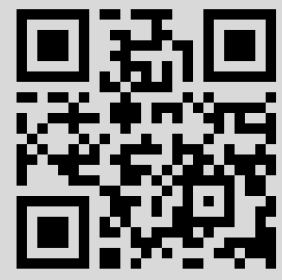




\section{Поведение нелинейного случайного процесса в окрестности его стационарных распределений}

\section{П. Н. Ярыкин}

Настоящая статья посвящена исследованию решения нелинейного стохастического дифференциального уравнения (СДУ)

$$
d \widetilde{X}_{t}=d W_{t}+\left(\int_{\mathbb{R}^{1}} b\left(\widetilde{X}_{t}, y\right) \mu_{t}^{\widetilde{X}}(d y)\right) d t, \quad t \geqslant 0,
$$

с начальным условием $\widetilde{X}_{0}=X_{0}$, где $W_{t}-$ броуновское движение, не зависящее от $X_{0}$, $\mu_{t}^{\widetilde{X}}$ - распределение $\widetilde{X}$ в момент времени $t, b\left(\widetilde{X}_{t}, y\right)$ - функция попарного симметричного взаимодействия, инвариантного относительно времени и сдвига:

$$
b(x, y)=-\beta(x-y)=-b(y, x), \quad \text { где } \quad \beta(x)=x+\alpha \sin x .
$$

Исторический обзор темы можно прочитать, например, в работе [1].

В работах [2] и [1] были доказаны существование и единственность решения при конечном $\mathrm{E} X_{0}$, существование и гладкость плотности $u(x, t)$ меры $\mu_{t}^{\widetilde{X}}$ относительно меры Лебега на $\mathbb{R}^{1}$ при $t>0$, сохранение первого момента (E $X_{t}=$ const). Там же было доказано, что любое стационарное решение уравнения (1) с нулевым первым моментом существует для любого $\alpha$ и имеет плотность вида

$$
p_{a}(x)=k_{a}^{-1} e^{-x^{2}+a \cos x} \quad \text { при } \quad a=2 \alpha \int \cos x p_{a}(x) d x .
$$

Более того, существуют $\widehat{\alpha}>1 / 2$ и $\widehat{a}<0$ такие, что: 1$)$ при $\alpha<0$ существует единственное стационарное решение, причем оно имеет вид $(3) ; 2)$ при $\alpha>\widehat{\alpha}$ существует не менее трех стационарных распределений вида (3) (с параметрами $a_{1}>0, a_{2}<\widehat{a}$ и $\left.\widehat{a} \leqslant a_{3} \leqslant 0\right)$.

Основная цель данной работы заключена в описании поведения процесса $\widetilde{X}_{t}$ при $t \rightarrow \infty$.

В силу трансляционной инвариантности системы каждая инвариантная мера порождает целый класс инвариантных мер, полученных из исходной сдвигом. Поэтому устойчивость стационарного распределения понимаем в следующем смысле.

ОПредЕление 1 . Стационарное решение $X_{\infty}$ уравнения (1) называется устойчивым, если существует слабая окрестность $U_{X_{\infty}}$ такая, что любое решение $\widetilde{X}_{t}$ уравнения (1) с начальным условием $X_{0} \in U_{X_{\infty}}$ таким, что $\mathrm{E} X_{0}=\mathrm{E} X_{\infty}$, слабо сходится к стационарному решению: $\widetilde{X}_{t} \underset{t \rightarrow \infty}{\stackrel{w}{\longrightarrow}} X_{\infty}$.

Заметим, что при наличии внешнего поля условие $\mathrm{E} X_{0}=\mathrm{E} X_{\infty}$ является лишним.

Функционал свободной энергии. Ключевым понятием этой работы является функционал свободной энергии $F(p)$. Положим

$$
F(p) \stackrel{\text { def }}{=} \frac{1}{2} \int p(x) \ln p(x) d x+\frac{1}{2} \iint \mathrm{B}(x-y) p(x) p(y) d x d y,
$$

если $p$ имеет плотность $p(x)$ и правая часть определена. Иначе положим $F(p) \stackrel{\text { def }}{=}+\infty$. Здесь $\mathrm{B}(x)=\frac{1}{2} x^{2}-\alpha \cos x-$ первообразная функции $\beta(x)$. Обратим внимание, что пространственный сдвиг меры $p(x) \rightarrow p(x-a)$ не изменяет значения функционала свободной энергии, что хорошо согласуется с отсутствием внешнего поля.

Сформулируем основные свойства функционала свободной энергии.

Лемма 1. а) $F(p)$ - полунепрерывная снизу функиия относительно слабой топологии пространства вероятностных мер, т.е. если $p_{n} \stackrel{w}{\longrightarrow} p_{0}, \operatorname{mo} F\left(p_{0}\right) \leqslant \varliminf_{n \rightarrow \infty} F\left(p_{n}\right)$.

б) Множество $K_{r}:=\{p \mid F(p) \leqslant r\}$ является секвенииально компактным относительно слабой топологии. 
Лемма 2. Пусть $u(x, t)$ - плотность решения СДУ (1), где $X_{0}$ имеет конечный четвертый момент. Тогда

$$
\frac{d}{d t} F(u(\cdot, t))=-\int\left(\frac{1}{2} u_{x}^{\prime}(x, t)+\int \beta(x-y) u(x, t) u(y, t) d y\right)^{2} \frac{1}{u(x, t)} d x \leqslant 0
$$

причем

$$
\frac{d}{d t} F(u(\cdot, t))=0 \Leftrightarrow u(\cdot, t)-\text { плотность стационарного распределения. }
$$

Таким образом, мы видим, что функционал свободной энергии не возрастает со временем и строго убывает в точках, не являющихся стационарными решениями уравнения нелинейного процесса (1). Значит, функционал (4) обладает основными свойствами функционалов свободной энергии, введенных другими авторами (см. [3], [4]).

Лемма 3. Пусть множество А является секвенциально компактным подмножеством пространства $\mathscr{P}(\mathbb{R})$ и $A$ таково, что если $\mu^{X_{0}} \in A$, то $u(\cdot, t) \in A \partial л я$ любого $t \geqslant 0$. Пусть также в множестве $A$ существует единственное стационарное распределение $p_{0}$.

Тогда $p_{0}$ является единственным минимумом функиионала свободной энергии $F$ на множестве $A$ и для любого $\mu^{X_{0}} \in A$ выполнено $u(\cdot, t) \underset{t \rightarrow \infty}{\stackrel{w}{\longrightarrow}} p_{0}$.

Леммы 1-3 являются основой доказательства предельных теорем.

\section{Предельное поведение решения.}

ТЕорема 1. При $\alpha \leqslant 1 / 2$ единственное стационарное решение СДУ (1) с заданным математическим ожиданием устойчиво, причем окрестностью сходимости $U_{X_{\infty}}$ является все множество вероятностных распределений $\mathscr{P}(\mathbb{R})$.

Поскольку класс четных распределений замкнут относительно процесса $\widetilde{X}_{t}$ ( $\widetilde{X}_{0}$ четно, значит, $\tilde{X}_{t}-$ четно) и уже содержит при больших положительных $\alpha$ три стационарных распределения, то ограничим рассмотрение этим классом.

Также получено, что $a_{1}$ и $a_{2}$ являются единственным решением предыдущего уравнения в указанных областях возможных значений. Более того, $a_{1} \sim 2 \alpha, a_{2} \sim-2 \alpha$ и существует $a_{0}<0$ такое, что $a_{3} \rightarrow a_{0}$ при $\alpha \rightarrow+\infty$. Следовательно,

$$
F\left(p_{a_{1}}\right)=-\frac{\alpha}{2}+O(\ln \alpha), \quad F\left(p_{a_{2}}\right)=-\frac{\alpha}{2}+O(\ln \alpha), \quad F\left(p_{a_{3}}\right) \geqslant F\left(p_{a_{0}}\right) .
$$

Теорема 2. Стационарное решение $p_{a_{3}}$ неустойчиво, т.е. для любой окрестности $U_{p_{a_{3}}}$ существует $p_{0} \in U_{p_{a_{3}}}$ такое, что решение СДУ (1) с началъным распределением $p_{0}$ не сходится $к$ стационарному решению с плотностью $p_{a_{3}}$.

Теорема 3. Стационарные распределения $p_{a_{1}}$ и $p_{a_{2}}$ являются устойчивыми в смысле определения 1.

Автор выражает благодарность научному руководителю А. Д. Маните за постоянное внимание к работе и М. А. Урусову за ценные советы.

\section{Список литературы}

[1] П. Н. Ярыкин, "Устойчивость нелинейного стохастического процесса, аппроксимирующего систему взаимодействующих частиц", Теория вероятн. и ее примен., 51:2 (2006) (в печати). [2] П.Н. Ярыкин, Вестн. Моск. ун-та. Сер. 1. Матем., мех., 2004, № 2, 58-61. [3] J. A. Carrillo, R. J. McCann, C. Villani, Rev. Mat. Iberoamericana, 19:3 (2003), 971-1018. [4] Y. Tamura, J. Fac. Sci. Univ. Tokyo Sect. IA Math., 31:1 (1984), 195-221.

П.Н. Ярыкин (P. N. Yarykin)

Московский государственный университет им. М. В. Ломоносова

E-mail: pavlik@plushka.msk.ru
Представлено А. В. Булинским Принято редколлегией 01.02.2006 